\title{
RASSEGNA
}

\section{Update sulla fibromialgia}

\section{Update on fibromyalgia}

\section{Fabiola Atzeni $^{\text {a,* }}$, Marco Cazzola ${ }^{\text {b }}$, Piercarlo Sarzi-Puttini ${ }^{\text {a }}$}

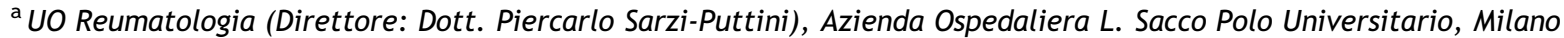 \\ ${ }^{\mathrm{b}}$ UO Medicina Riabilitativa, Azienda Ospedaliera Ospedale di Circolo di Busto Arsizio, PO di Saronno (VA)
}

Ricevuto il 30 aprile 2010; accettato il 6 dicembre 2010

disponibile online il 19 maggio 2011

\section{KEYWORDS \\ Fibromyalgia; \\ Diffuse pain; \\ Pharmacological \\ therapy; \\ Physiotherapy.}

\begin{abstract}
Summary
Introduction: Fibromyalgia (FM) is a chronic pain syndrome characterized by widespread pain, fatigue, sleep alterations, and distress. It affects at least $2 \%$ of the adult population. The etiology of FM is not completely understood, and the syndrome is influenced by stress, physical illness, and a variety of pain conditions. Emerging evidence indicates that augmented pain processing within the central nervous system plays a primary role in the pathophysiology of this disorder. Diagnosis may be difficult because of the multifaceted nature of the syndrome and its overlap with other chronically painful conditions. This article reviews the most recent data in the literature on FM. Materials and methods: There are currently no instrumental tests or specific diagnostic markers for FM. In fact, many of the existing indicators are regarded as significant for research purposes only.

Results: Differential diagnosis requires an extensive clinical examination and complete patient history. Chest-X-rays and abdominal ultrasonography are the first steps in the general evaluation of a patient with suspected FM.

Conclusions: A variety of pharmacological treatments have been used to treat FM, including antidepressants, nonsteroidal anti-inflammatory drugs, opioids, sedatives, muscle relaxants, and antiepileptics. Physical exercise and multimodal cognitive-behavioral therapy seem to be the most widely accepted and beneficial forms of non-pharmacological therapy.

(c) 2011 Elsevier Srl. All rights reserved.
\end{abstract}

\section{Introduzione}

La fibromialgia (FM) o sindrome fibromialgica è una patologia caratterizzata da dolorabilità muscolo-scheletrica diffusa e astenia, che colpisce approssimativamente 1,5-2 milioni di italiani [1,2]. La FM è una patologia caratteristica dell'età media con una prevalenza maggiore tra i 20 e i 50 anni, ma si può comunque osservare anche in età giovanile o dopo i 60 anni. Le donne sono più colpite degli uomini, con un rapporto di 9:1 [1,2].

\footnotetext{
* Corrispondenza: UO Reumatologia, Azienda Ospedaliera L. Sacco, via G.B. Grassi 74 - 20157 Milano.

E-mail: atzenifabiola@hotmail.com (F. Atzeni).
} 
Questa condizione viene definita "sindrome" poiché esistono segni e sintomi clinici che sono contemporaneamente presenti. Spesso può essere confusa con altre malattie poiché alcuni dei suoi tratti possono essere riscontrati in altre situazioni cliniche, ma sebbene possa assomigliare a una patologia articolare non si tratta di artrite e non causa deformità delle strutture articolari.

La FM è classificata dalla Società Italiana di Reumatologia tra i reumatismi extrarticolari o dei tessuti molli ma, in realtà, non sono mai state riscontrate alterazioni morfologiche a carico delle sedi in cui il dolore è percepito dai pazienti (muscoli, tendini, entesi). Più recentemente la FM è stata considerata una sindrome da sensibilizzazione centrale la cui base fisiopatologica andrebbe ricercata in un'eccessiva sensibilità delle strutture nervose deputate alla percezione, trasmissione e processazione degli input nocicettivi.

Fino a pochi mesi fa la diagnosi era effettuata sulla base dei criteri classificativi proposti dall'American College of Rheumatology (ACR) nel 1990 [3] che richiedono la presenza, da almeno 3 mesi, di dolore muscolo-scheletrico diffuso e la positività di almeno 11 dei 18 tender point (TP), evocabili alla digitopressione indotta da una pressione di $4 \mathrm{~kg} / \mathrm{cm}^{2} 0$ mediante algometro a pressione posizionato in sedi codificate (fig. 1). Più recentemente sono stati proposti criteri diagnostici che non richiedono la ricerca dei TP per porre la diagnosi [4] (tab. 1).

Oltre al dolore cronico diffuso i pazienti affetti da FM riferiscono molteplici sintomi extrascheletrici tra i quali i più frequenti sono depressione, cefalea, alterazioni del sonno, astenia, rigidità mattutina, parestesie agli arti, toracoalgie atipiche, colon e vescica irritabili. Questi sintomi sono influenzati da fattori quali le condizioni climatiche, l'attività fisica, gli stress psicofisici e i disturbi del sonno. In circa la metà dei pazienti i sintomi appaiono dopo una sindrome similinfluenzale o uno stress di tipo fisico o psichico. Circa il 30\% dei pazienti fibromialgici presenta disturbi psicologici, in prevalenza di tipo ansioso-depressivo. Tuttavia, il dolore è il sintomo predominante e, generalmente, si manifesta in tutto il corpo, sebbene possa iniziare in una sede localizzata, come il rachide cervicale e le spalle, e successivamente diffondersi in altre sedi con il trascorrere degli anni [1].

\section{Eziologia e patogenesi}

L'eziologia della FM non è ancora stata completamente chiarita, ma si pensa che alla sua insorgenza concorrano fattori esterni, quali lo stress, altre malattie e una varietà di condizioni dolorose croniche diverse in alcuni pazienti (ma non in tutti).

La patogenesi riconoscerebbe, invece, alterazioni a carico di numerosi neurotrasmettitori e del sistema neuroendocrino: le modificazioni che si ritiene rivestano un maggior ruolo nell'insorgenza della malattia riguardano la riduzione dei livelli di ammine biogene, un'aumentata concentrazione di neurotrasmettitori eccitatori (tra cui la sostanza P) e una disregolazione dell'asse ipotalamo-ipofisi-surrene [5-10]. La sensibilizzazione neuronale in diverse aree del sistema nervoso centrale rappresenta, ultimamente, l'ipotesi patogenetica maggiormente accreditata. Si pensa che l'insorgenza e la cronicizzazione della malattia siano dovute all'interazione tra un'aberrazione dei meccanismi fisiologici alla base della

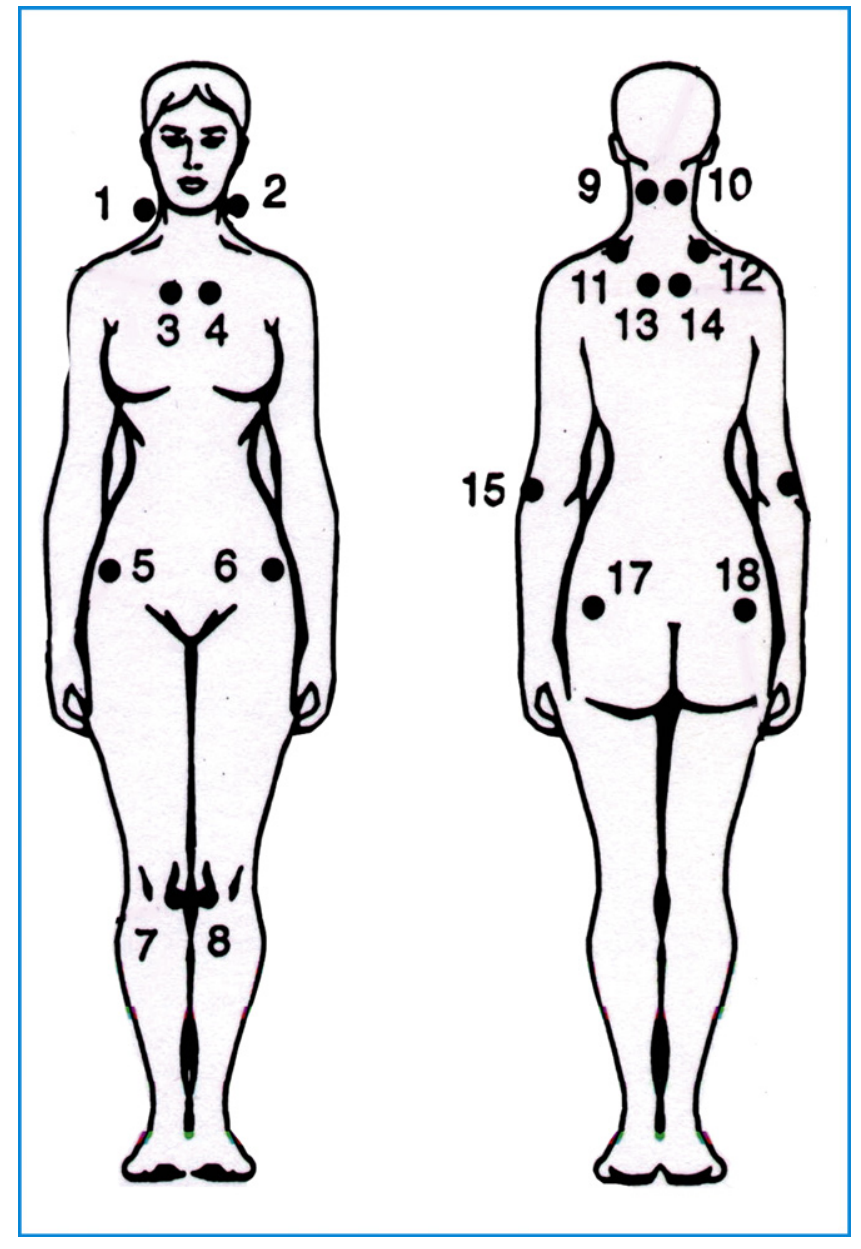

Figura 1 Distribuzione dei tender point (fonte: modificata da http: //www.healthstudio.com).

nocicezione e molteplici fattori psicologici e ambientali [11-14] (tab. 2).

Recentemente, facendo riferimento al principale meccanismo patogenetico identificato come causa della sintomatologia, scheletrica ed extrascheletrica, della FM e di altre sindromi definite "disfunzionali", sembra corretto includere la FM nell'ambito delle sindromi da sensibilizzazione centrale e definirla come segue: "La fibromialgia è una sindrome da sensibilizzazione centrale caratterizzata dalla disfunzione dei neurocircuiti preposti alla percezione, trasmissione e processazione delle afferenze nocicettive, con prevalente estrinsecazione del dolore a livello dell'apparato muscoloscheletrico. Oltre al dolore possono essere presenti molteplici sintomi di accompagnamento (astenia, disturbi del sonno, dolori addominali...) comuni ad altre sindromi da sensibilizzazione centrale. Particolari caratteristiche genetiche e una ridotta capacità individuale di tolleranza agli stressor predispongono all'insorgenza della malattia" [1].

\section{Sintomi ed esame obiettivo}

La presenza, da almeno 3 mesi, di dolore muscolo-scheletrico diffuso rappresenta il sintomo patognomonico della FM [1]. 
Tabella 1 Criteri diagnostici e valutazione della sindrome fibromialgica.

\section{Criteri}

Un paziente soddisfa i criteri diagnostici per la fibromialgia se risponde alle 3 seguenti condizioni:

1. Indice per il dolore diffuso (WPI) $\geq 7$ e il punteggio alla scala della severità dei sintomi (SS) $\geq 5$ o WPI 3-6 e punteggio alla scala della SS $\geq 9$

2. I sintomi sono presenti con la stessa intensità da almeno 3 mesi

3. Il paziente non ha una patologia che potrebbe spiegare in modo diverso il dolore

Valutazione

1. WPI: sommare il numero delle aree nelle quali il paziente ha accusato dolore durante l'ultima settimana.

In quante aree il paziente ha avuto dolore?

Il punteggio dovrebbe essere compreso tra 0 e 19

\begin{tabular}{lll} 
Cingolo scapolare, sinistro & Anca (gluteo, trocantere), sinistra & Mascella, sinistra \\
\hline Cingolo scapolare, destro & Anca (gluteo, trocantere), destra & Mascella, destra \\
\hline Braccio, sinistro & Coscia, sinistra & Torace \\
\hline Braccio, destro & Coscia, destra & Addome \\
\hline Avambraccio, sinistro & Gamba, sinistra & Area dorsale \\
\hline Avambraccio, destro & Gamba, destra & Area lombare \\
\hline & & Collo
\end{tabular}

2. Punteggio alla scala della severità dei sintomi (SS)

- Astenia

- Svegliarsi non riposati

- Disturbi cognitivi

Per ognuno dei 3 sintomi suddetti, indicare il grado di severità durante l'ultima settimana utilizzando la seguente scala:

$0=$ Nessun problema

1 = Problemi lievi o moderati, generalmente limitati o intermittenti

2 = Problemi moderati, considerevoli, spesso presenti e/o a moderata intensità

3 = Problemi severi, penetranti, continui, che compromettono la vita

$0=$ Nessun sintomo

$1=$ Pochi sintomi

$2=$ Un modesto numero di sintomi

$3=$ Una gran quantità di sintomi

Il punteggio alla scala della SS è fornito dalla somma della severità dei 3 sintomi (astenia, svegliarsi non riposati, disturbi cognitivi) più l'estensione (severità) dei sintomi somatici in generale

Il punteggio finale è compreso tra 0-12

* I sintomi somatici che devono essere presi in considerazione sono: dolore muscolare, sindrome del colon irritabile, fatica/affaticamento, problemi di ideazione o di memoria, debolezza muscolare, cefalea, dolori/crampi addominali, intorpidimento/formicolio, vertigini, insonnia, depressione, stipsi, dolore all'addome superiore, nausea, irritabilità, dolore al petto, vista annebbiata, febbre, diarrea, secchezza delle fauci, prurito, sensazione di bolo, fenomeno di Raynaud, orticaria/angiodema, tinnito, vomito, pirosi, ulcere orali, perdita o cambiamento del gusto, attacco epilettico, secchezza degli occhi, respiro corto, perdita dell'appetito, rash, fotosensibilità, perdita dell'udito, facile presenza di lividi, perdita dei capelli, minzione frequente, minzione dolorosa e spasmi vescicali.

Il dolore fibromialgico, tipicamente, è diffuso o multifocale, di intensità variabile nel corso della giornata, talora migrante, quanto meno per intensità, nelle diverse sedi corporee; vari fattori esterni, fisici e/o psichici, ne influenzano le riacutizzazioni. Queste caratteristiche sono quelle del dolore centrale, differenti rispetto a quelle di un dolore periferico in cui sia la localizzazione sia l'intensità sono molto più costanti. I pazienti, talora, percepiscono come dolorosi stimoli solitamente innocui, per esempio indossare gli abiti; se interrogati adeguatamente, non di rado riferiscono parestesie o disestesie associate al dolore [1].
Oltre al dolore possono coesistere numerosi sintomi, apparentemente non correlati. L'astenia, i disturbi del sonno, la debolezza, la labilità emotiva e i deficit di memoria, l'intolleranza al caldo o al freddo, i disturbi visivi e uditivi, i sintomi vestibolari, la sensazione di secchezza a livello delle mucose e le variazioni non giustificate del peso corporeo sono solo i più frequenti. I fenomeni "allergici", quali riniti, sinusiti, senso di congestione nasale e sintomi a carico delle basse vie respiratorie, sono riportati più frequentemente dai pazienti fibromialgici rispetto ai controlli, anche se, in realtà, si tratta quasi sempre di fenomeni di 
Tabella 2 Fattori di rischio che predicono l'insorgenza e la persistenza del dolore muscolo-scheletrico diffuso.

\begin{tabular}{l}
\hline Demografici \\
\hline Psicologici \\
\hline Sociali \\
\hline Storia familiare positiva di dolore cronico \\
\hline Basso livello di scolarità \\
\hline Mancanza di supporto sociale ed economico \\
\hline
\end{tabular}

ipersensibilità piuttosto che di reazioni immunitarie lgEmediate $[1,2]$. Nelle donne le comorbilità più frequenti sono la dismenorrea, la cistite interstiziale, la vestibolite e la vulvodinia, nell'uomo, invece, la prostatite non batterica.

L'esame obiettivo articolare è solitamente normale, non essendo riscontrabili limitazioni funzionali o segni obiettivi di flogosi a carico delle articolazioni, anche quando sono sede di dolore spontaneo [15]. La presenza di punti elettivamente dolenti alla pressione, TP, rappresenta il segno obiettivo più caratteristico della FM. I TP possono essere definiti come aree iperalgesiche situate in corrispondenza di specifiche sedi muscolari e tendinee; alcune di queste zone hanno una distribuzione topografica simile a quella di alcune sindromi dolorose extrarticolari localizzate, come l'epicondilite e la tendinite trocanterica.

\section{Esami di laboratorio e diagnosi differenziale}

La diagnosi di FM comporta, in primo luogo, l'esclusione di qualsiasi altra causa di dolore articolare e muscolare. Gli esami di laboratorio in genere non sono utili, se non per la diagnosi differenziale [15].

Un criterio per decidere quali e quante indagini di laboratorio eseguire è rappresentato dalla durata di malattia: se risale a diversi anni prima è possibile limitare notevolmente le indagini, mentre nelle forme di recente insorgenza possono essere necessarie più indagini per un inquadramento diagnostico corretto (tab. 3). Altre indagini più sofisticate

Tabella 3 Esami di laboratorio consigliati.

\begin{tabular}{lc}
\hline $\begin{array}{l}\text { Insorgenza dei } \\
\text { sintomi }<12 \text { mesi }\end{array}$ & $\begin{array}{c}\text { Insorgenza dei } \\
\text { sintomi }>12 \text { mesi }\end{array}$ \\
\hline VES & VES \\
\hline PCR & Emocromo \\
\hline Emocromo & TSH \\
\hline ANA & \\
\hline CPK & \\
\hline TSH & \\
\hline
\end{tabular}

Funzionalità epatica e renale

Legenda: $V E S=$ velocità di eritrosedimentazione; $P C R=$ proteina C-reattiva; ANA = anticorpi antinucleo; $\mathrm{CPK}=$ creatinfosfochinasi; $\mathrm{TSH}=$ ormone tireostimolante. non sono solitamente necessarie, a meno che siano non indicate in base all'esame obiettivo e allo screening di laboratorio.

Molti dei sintomi, scheletrici ed extrascheletrici, caratteristici della FM possono essere riscontrati in numerose malattie, pertanto riveste fondamentale importanza la diagnosi differenziale con altre cause di dolore cronico. La FM, inoltre, può coesistere in associazione con patologie diverse, reumatiche e non reumatiche (FM concomitante), inducendo errori diagnostici. Numerose malattie reumatiche autoimmuni come il lupus eritematoso sistemico, la sindrome di Sjögren, altre connettiviti sistemiche, l'artrite reumatoide, le spondiloartriti e malattie non reumatologiche quali l'ipotiroidismo, l'anemia, la malattia di Lyme, l'infezione da virus dell'epatite C, la sindrome da fatica cronica (SFC) e le neoplasie occulte sono possibili cause di algie a carico dell'apparato muscolo-scheletrico e di astenia intensa [15] (tab. 4).

La diagnosi differenziale tra lupus eritematoso sistemico e FM può rappresentare un dilemma clinico poiché i pazienti affetti da queste due malattie possono lamentare molti sintomi comuni. Oltre al dolore, all'astenia e alla rigidità, infatti, in entrambi i casi possono essere presenti fenomeno di Raynaud, sindrome sicca, alterazioni cognitive e deflessione del tono dell'umore [16-18]. La diagnosi differenziale è oltremodo difficile in quei pazienti con FM che presentano positività agli anticorpi antinucleo (ANA); la positività degli ANA a basso titolo, infatti, è stata segnalata nei pazienti fibromialgici in assenza di segni clinici di connettivite sistemica [19]. In letteratura la FM è considerata, talora, la modalità d'esordio della sindrome di Sjögren, anche se le evidenze sono piuttosto controverse [20]. Nei pazienti con FM che tra $\mathrm{i}$ disturbi extrascheletrici lamentano la sindrome sicca dovrebbero essere eseguite, a fini diagnostico-differenziali, ulteriori indagini come il test di Schirmer, la capillaroscopia, la biopsia delle ghiandole salivari minori e la determinazione del pattern autoanticorpale. Probabilmente i pazienti con sindrome di Sjögren hanno una FM secondaria, i cui sintomi predominanti hanno portato a formulare la diagnosi di FM prima di quella di sindrome di Sjögren. Molte altre malattie, reumatiche e non reumatiche, possono essere confuse con la FM [21]. La polimialgia reumatica è caratterizzata da dolore diffuso, rigidità e incremento della velocità di eritrosedimentazione (VES), ma nel $20 \%$ dei casi la VES può essere normale [22].

Le miopatie infiammatorie e l'osteomalacia possono presentarsi con sintomi clinici simili a quelli della FM, ma le

Tabella 4 Condizioni che simulano la fibromialgia.

\begin{tabular}{ll}
\hline Comuni & \multicolumn{1}{c}{ Meno frequenti } \\
\hline Ipotiroidismo & Epatite C \\
\hline Polimialgia reumatica & $\begin{array}{l}\text { Sindrome delle apnee } \\
\text { notturne }\end{array}$ \\
\hline $\begin{array}{l}\text { Esordio di alcune malattie } \\
\text { reumatiche (per esempio AR, LES) }\end{array}$ & Malformazione di Chiari \\
\hline Sindrome di Sjögren & \\
\hline $\begin{array}{l}\text { Legenda: AR = artrite reumatoide; } \\
\text { sistemico. }\end{array}$ & LES = lupus eritematoso
\end{tabular}


indagini strumentali e di laboratorio (radiologia, creatinfosfochinasi, ipofosfatemia, biopsia muscolare) permettono di formulare una diagnosi corretta [23].

Le alterazioni della funzione tiroidea possono esordire con profondo affaticamento, debolezza muscolare e dolorabilità diffusa [24].

Tra SFC e FM esistono numerose similitudini cliniche e, forse, patogenetiche; oltre il 70\% dei pazienti fibromialgici lamenta gli stessi sintomi caratteristici della SFC, tant'è vero che possono rispettare i criteri diagnostici formulati per entrambe queste condizioni [25] (tab. 5). È probabile che SFC e FM altro non siano che la stessa sindrome in cui i sintomi prevalenti, e maggiormente invalidanti per il paziente, sono rappresentati, rispettivamente, dalla stanchezza o dal dolore.

La prevalenza della FM nei pazienti portatori di infezione da HCV è compresa, nei diversi lavori che hanno studiato questa possibile associazione, tra il 5 e il 19\%, ma non vi è accordo circa il possibile ruolo patogenetico del virus [26].

\section{Terapia}

La FM è una sindrome complessa che comporta significative limitazioni funzionali, peggioramento della qualità di vita e costi sociali considerevoli; il trattamento appropriato, tuttavia, sembra ridurre in modo significativo il grado di disabilità [28] (tab. 6).

Nessun trattamento è uniformemente efficace in tutti i pazienti; le strategie terapeutiche, che consistono in trattamenti farmacologici e non farmacologici, devono essere personalizzate per il singolo paziente e il clinico può avere la necessità di provare differenti modalità terapeutiche prima di ottenere un miglioramento ottimale dei sintomi del paziente [27-29]. Ne consegue che l'approccio terapeutico è andato strutturandosi in modo articolato avvalendosi sia di presidi farmacologici capaci di interferire con le anomalie descritte, sia di modalità terapeutiche non farmacologiche (biofeedback, ipnositerapia, fitness cardiovascolare, terapia cognitiva comportamentale, elettroagopuntura ecc.) [29-31]. Un approccio integrato di tipo comportamentale, farmacologico e non farmacologico può in molti casi apportare notevoli benefici; risulta tuttavia importante, oltre a stabilire la diagnosi di SF, definire le condizioni fisiche e psicologiche del paziente stesso, educarlo alla malattia e formulare un piano terapeutico con obiettivi ragionevoli da raggiungere [27-31].

\section{Terapia farmacologica e non farmacologica}

I farmaci antinfiammatori utilizzati per trattare molte patologie reumatiche non mostrano importanti effetti nella FM [32]. Tuttavia, basse dosi di acido acetilsalicilico, l'ibuprofene e il paracetamolo possono recare qualche sollievo al dolore nelle forme di FM associata, per esempio, a osteoartrosi. Il tramadolo, un agonista debole per i recettori $\mu$ per gli oppioidi, un farmaco analgesico centrale, può ridurre la sintomatologia dolorosa del paziente con FM. I cortisonici sono inefficaci e dovrebbero essere evitati per i loro potenziali effetti collaterali [33]. I farmaci che favoriscono il sonno profondo e il rilassamento muscolare aiutano molti pazienti affetti da FM a riposare meglio. Questi farmaci comprendono gli antidepressivi triciclici (amitriptilina ecc.) e gli inibitori selettivi del reuptake della serotonina (paroxetina, fluoxetina ecc.) e altri farmaci ad azione prevalentemente miorilassante, ma simili strutturalmente agli antidepressivi (ciclobenzaprina) [27,33]. Sebbene abbiano come principale indicazione la depressione, essi vengono abitualmente prescritti a bassi dosaggi ai pazienti affetti da FM, di solito prima di andare a letto. Nei pazienti con FM, sono principalmente utilizzati per lenire il dolore, rilassare $i$ muscoli e migliorare la qualità del sonno anziché per il loro effetto antidepressivo. Gli anticonvulsivanti, come il gabapentin e il pregabalin, sono molecole efficaci nel ridurre $i$ sintomi della FM [7]. Nei pazienti che non rispondono alla monoterapia, alcuni di questi farmaci possono essere utilizzati in combinazione [33].

Tabella 5 Criteri diagnostici per la sindrome da fatica cronica.

Condizione di affaticamento cronico, accertata clinicamente, persistente, recidivante e non spiegabile:

- a esordio recente o comunque collocabile temporalmente

- non risultante da un periodo di sforzi in corso

- non alleviata dal riposo

- invalidante dal punto di vista occupazionale, sociale o personale

Presenza concomitante di almeno 4 dei seguenti sintomi, ciascuno dei quali deve essere stato presente continuativamente

o essersi ripresentato saltuariamente per almeno 6 mesi, non predominante rispetto alla fatica:

- deterioramento, riferito dal paziente, della memoria a breve termine, sufficientemente severo da determinare una riduzione degli standard precedenti nelle attività lavorative, scolastiche, sociali o personali

- faringodinia

- linfoadenopatia cervicale o ascellare

- dolore muscolare

- dolore poliarticolare senza tumefazione o arrossamento

- cefalee con caratteristiche nuove o di gravità diversa

- sonno non ristoratore

- malessere insorto dopo sforzi, che dura più di 24 ore

Fonte: modificata da Fukuda K, et al. Ann Intern Med 1994;121(12):953-9. 
Tabella 6 Efficacia di alcuni farmaci sui sintomi principali della fibromialgia.

\begin{tabular}{lcccc}
\hline & Dolore & Sonno & Astenia & Umore \\
\hline Triciclici & + & + & + & - \\
\hline SSRI & \pm & \pm & \pm & \pm \\
\hline SNRI & + & - & + & + \\
\hline IMAO & \pm & \pm & \pm & \pm \\
\hline FANS & - & - & - & - \\
\hline Antiepilettici & + & + & + & - \\
\hline Sedativi/ipnotici & - & + & - & - \\
\hline Oppioidi & + & + & - & - \\
\hline Miorilassanti & & & \pm & \\
\hline Antagonisti NMDA & & & & \\
\hline
\end{tabular}

Legenda: SSRI = inibitori selettivi del reuptake della serotonina; SNRI = inibitori del reuptake della serotonina e della noradrenalina; $I M A O=$ inibitori delle monoamminossidasi; FANS = farmaci antinfiammatori non steroidei. J Reumathol 1999:26:408-12.

Poche molecole, tuttavia, si sono dimostrate utili quando valutate in studi randomizzati controllati; tra di esse, quelle testate più recentemente sono la duloxetina e il milnacipran, in grado di inibire il reupkake sia della serotonina sia della noradrenalina, e il pregabalin, che appartiene alla classe degli antiepilettici $[6,32,33]$ (tab. 7). Nessuno dei farmaci menzionati e utilizzati è attualmente approvato dalla European Medicines Agency (EMEA) per il trattamento della FM, mentre il pregabalin, il milnacipram e la duloxetina sono stati approvati dalla Food and Drug Administration (FDA) per il trattamento della FM negli Stati Uniti.

Nonostante la migliore comprensione dei meccanismi patogenetici alla base di questa malattia, tuttavia, i risultati ottenuti con il solo trattamento farmacologico sono spesso deludenti e alcuni specialisti ritengono che non esista una terapia realmente efficace $[27,33]$.

Il trattamento non farmacologico comprende le tecniche di stiramento muscolare e di allenamento dei muscoli dolenti e l'incremento graduale del fitness cardiovascolare (aerobico). Attività aerobica a basso o nullo impatto, come camminare, andare in bicicletta, nuotare o praticare la ginnastica in acqua sono generalmente il modo migliore di iniziare un programma di esercizi e di migliorare la forma fisica [34]. È utile inoltre consultare un terapista della riabilitazione che aiuti a stabilire uno specifico programma di esercizi per migliorare la postura, la flessibilità e la forma fisica. Anche le terapie cosiddette non convenzionali quali gli integratori dietetici o i trattamenti non farmacologici come il biofeedback, l'agopuntura, la ginnastica dolce e lo yoga possono avere effetti positivi sui sintomi del paziente FM [34].

Tabella 7 Farmaci antidepressivi e antiepilettici: effetti indesiderati e controindicazioni.

\begin{tabular}{|c|c|}
\hline Farmaco & Effetti indesiderati, controindicazioni e commenti \\
\hline & Antidepressivi \\
\hline Antidepressivi triciclici & $\begin{array}{l}\text { Effetti indesiderati: secchezza delle fauci, stipsi, ritenzione urinaria, sedazione, } \\
\text { aumento ponderale } \\
\text { Controindicazioni: alterazioni della conduzione cardiaca, recenti patologie cardiache } \\
\text { significative, glaucoma ad angolo chiuso }\end{array}$ \\
\hline Amitriptilina, imipramina & $\begin{array}{l}\text { La ammine terziarie hanno maggiori effetti anticolinergici e, perciò, non dovrebbero } \\
\text { essere utilizzate nei pazienti anziani }\end{array}$ \\
\hline \multirow[t]{2}{*}{ Desipramina, nortriptilina } & La ammine secondarie hanno minori effetti indesiderati anticolinergici \\
\hline & Inibitori selettivi del reuptake della serotonina (SSRI) \\
\hline \multirow[t]{2}{*}{ Fluoxetina, paroxetina } & $\begin{array}{l}\text { Effetti indesiderati: nausea, sedazione, riduzione della libido, disfunzioni sessuali, } \\
\text { cefalea, aumento ponderale } \\
\text { L'efficacia nelle sindromi dolorose croniche è relativamente scarsa }\end{array}$ \\
\hline & Inibitori del reuptake della serotonina e della noradrenalina (SNRI) \\
\hline $\begin{array}{l}\text { Milnacipran (non in } \\
\text { vendita in Italia) }\end{array}$ & $\begin{array}{l}\text { Effetti indesiderati: ansia, sudorazione, vertigini, disuria, secchezza delle fauci, stipsi, } \\
\text { palpitazioni }\end{array}$ \\
\hline Venlafaxina & $\begin{array}{l}\text { Effetti indesiderati: cefalea, nausea, sudorazione, sedazione, ipertensione, convulsioni } \\
\text { Effetti serotoninergici a dosi }<150 \mathrm{mg} / \text { die; effetti misti, serotoninergici e noradrenergici, } \\
\text { a dosaggi maggiori }\end{array}$ \\
\hline \multirow[t]{2}{*}{ Duloxetina } & Effetti indesiderati: nausea, secchezza delle fauci, stipsi, vertigini, insonnia \\
\hline & Farmaci antiepilettici \\
\hline Gabapentin & Effetti indesiderati: sonnolenza, vertigini, astenia, nausea, sedazione, aumento ponderale \\
\hline Pregabalin & Effetti indesiderati: sonnolenza, vertigini, astenia, nausea, sedazione, aumento ponderale \\
\hline
\end{tabular}




\section{Conflitto di interesse}

Gli autori dichiarano di essere esenti da conflitto di interessi.

\section{Bibliografia}

[1] Cazzola M, Sarzi Puttini P, Stisi S, Di Franco M, Bazzichi L, Carignola R, et al., Italian Fibromyalgia Network. Fibromyalgia syndrome: definition and diagnostic aspects. Reumatismo 2008;60(Suppl 1):3-14.

[2] Sarzi-Puttini P, Atzeni F. La sindrome fibromialgica. In: Maddali Bongi S (a cura di). Riabilitazione reumatologica. Approccio multidisciplinare. Milano: Edra; 2007. p. 979-94.

[3] Wolfe F, Smythe HA, Yunus MB, Bennett RM, Bombardier C, Goldenberg DL, et al. The American College of Rheumatology 1990 Criteria for the Classification of Fibromyalgia. Report of the Multicenter Criteria Committee. Arthritis Rheum 1990; 33(2):160-72.

[4] Wolfe F. New American College of Rheumatology criteria for fibromyalgia: a twenty-year journey. Arthritis Care Res (Hoboken) 2010;62(5):583-4.

[5] Benjamin S, Morris S, McBeth J, Macfarlane GJ, Silman AJ. The association between chronic widespread pain and mental disorder: a population-based study. Arthritis Rheum 2000;43(3): 561-7.

[6] Bennett RM. Adult growth hormone deficiency in patients with fibromyalgia. Curr Rheumatol Rep 2002;4(4):306-12.

[7] Sarzi-Puttini P, Atzeni F, Cazzola M. Neuroendocrine therapy of fibromyalgia syndrome: an update. Ann N Y Acad Sci 2010; 1193:91-7.

[8] Sarzi-Puttini P, Atzeni F, Diana A, Doria A, Furlan R. Increased neural sympathetic activation in fibromyalgia syndrome. Ann $\mathrm{N}$ Y Acad Sci 2006;1069:109-17.

[9] Rizzi M, Sarzi-Puttini P, Atzeni F, Capsoni F, Andreoli A, Pecis M, et al. Cyclic alternating pattern: a new marker of sleep alteration in patients with fibromyalgia? J Rheumatol 2004;31(6):1193-9.

[10] Furlan R, Colombo S, Perego F, Atzeni F, Diana A, Barbic F, et al. Abnormalities of cardiovascular neural control and reduced orthostatic tolerance in patients with primary fibromyalgia. J Rheumatol 2005;32(9):1787-93.

[11] Staud R, Smitherman ML. Peripheral and central sensitization in fibromyalgia: pathogenetic role. Curr Pain Headache Rep 2002;6(4):259-66.

[12] Yunus MB. Fibromyalgia and overlapping disorders: the unifying concept of central sensitivity syndromes. Semin Arthritis Rheum 2007;36(6):339-56.

[13] Winfield JB. Fibromyalgia and related central sensitivity syndromes: twenty-five years of progress. Semin Arthritis Rheum 2007;36(6):335-8.

[14] Yunus MB. Central sensitivity syndromes: a new paradigm and group nosology for fibromyalgia and overlapping conditions, and the related issue of disease versus illness. Semin Arthritis Rheum 2008;37(6):339-52.

[15] Atzeni F, Salaffi F, Bazzichi L, Gracely RH, Carignola R, Torta R, et al., Italian Fibromyalgia Network. The evaluation of the fibromyalgia patients. Reumatismo 2008;60(Suppl 1):36-49.
[16] Martínez-Lavín M. Overlap of fibromyalgia with other medical conditions. Curr Pain Headache Rep 2001;5(4):347-50.

[17] Bennett R. The concurrence of lupus and fibromyalgia: implications for diagnosis and management. Lupus 1997;6(6):494-9.

[18] Akkasilpa S, Goldman D, Magder LS, Petri M. Number of fibromyalgia tender points is associated with health status in patients with systemic lupus erythematosus. J Rheumatol 2005;32(1):48-50.

[19] Buskila D, Press J, Abu-Shakra M. Fibromyalgia in systemic lupus erythematosus: prevalence and clinical implications. Clin Rev Allergy Immunol 2003;25(1):25-8.

[20] Bonafede RP, Downey DC, Bennett RM. An association of fibromyalgia with primary Sjögren's syndrome: a prospective study of 72 patients. J Rheumatol 1995;22(1):133-6.

[21] Wolfe F, Hawley DJ, Wilson K. The prevalence and meaning of fatigue in rheumatic disease. J Rheumatol 1996;23(8):1407-17.

[22] Proven A, Gabriel SE, O'Fallon WM, Hunder GG. Polymyalgia rheumatica with low erythrocyte sedimentation rate at diagnosis. J Rheumatol 1999;26(6):1333-7.

[23] Reginato AJ, Falasca GF, Pappu R, Mcknight B, Agha A. Musculoskeletal manifestations of osteomalacia: report of 26 cases and literature review. Semin Arthritis Rheum 1999;28(5): 287-304.

[24] Bazzichi L, Rossi A, Giuliano T, De Feo F, Giacomelli C, Consensi A, et al. Association between thyroid autoimmunity and fibromyalgic disease severity. Clin Rheumatol 2007;26(12): 2115-20.

[25] International Chronic Fatigue Syndrome Study Group, Fukuda K, Straus SE, Hickie I, Sharpe MC, Dobbins JG, Komaroff A. The chronic fatigue syndrome: a comprehensive approach to its definition and study. Ann Intern Med 1994;121(12):953-9.

[26] Lormeau C, Falgarone G, Roulot D, Boissier MC. Rheumatologic manifestations of chronic hepatitis $C$ infection. Joint Bone Spine 2006;73(6):633-8.

[27] Sarzi-Puttini P, Buskila D, Carrabba M, Doria A, Atzeni F. Treatment strategy in fibromyalgia syndrome: where are we now? Semin Arthritis Rheum 2008;37(6):353-65.

[28] Burckhardt CS. Multidisciplinary approaches for management of fibromyalgia. Curr Pharm Des 2006;12(1):59-66.

[29] Keel P. Pain management strategies and team approach. Baillieres Best Pract Res Clin Rheumatol 1999;13(3):493-506.

[30] Oliver K, Cronan TA, Walen HR. A review of multidisciplinary interventions for fibromyalgia patients: where do we go from here? J Musculoskel Pain 2001;9:63-80.

[31] Turk DC, Okifuji A, Sinclair JD, Starz TW. Interdisciplinary treatment for fibromyalgia syndrome: clinical and statistical significance. Arthritis Care Res 1998;11(3):186-95.

[32] Cazzola M, Sarzi-Puttini P, Buskila D, Atzeni F. Pharmacological treatment of fibromyalgia. Reumatismo 2007;59(4):280-91.

[33] Sarzi-Puttini P, Torta R, Marinangeli F, Biasi G, Spath M, Buskila D, et al., Italian Fibromyalgia Network. Fibromyalgia syndrome: the pharmacological treatment options. Reumatismo 2008; 60(Suppl 1):50-8.

[34] Casale R, Cazzola M, Arioli G, Gracely RH, Ceccherelli F, Atzeni F, et al., Italian Fibromyalgia Network. Non pharmacological treatments in fibromyalgia. Reumatismo 2008; 60(Suppl 1):59-69. 